\title{
DIELECTRIC PROPERTIES AND ELECTRON SPECTRUM OF THE MÜLLER MODEL IN THE HIGH-TEMPERATURE SUPERCONDUCTIVITY THEORY
}

\author{
I.V. Stasyuk and A.M. Shvaika \\ Institute for Condensed Matter Physics, Ukrainian Academy of Sciences \\ Svientsitsky St. 1, Lviv 290011, Ukraine
}

(Received Oclober 5, 1992; revised version April 30, 1993)

\begin{abstract}
The paper is devoted to the investigation of the single-electron excitation spectrum, effective exchange interaction and dielectric susceptibility of the Müller model for high-temperature superconductivity. It is shown that the interaction with the anharmonic vibration mode leads to the additional splitting of the electron bands and thcir narrowing. The sign of the effective exchange interaction between electrons depends on the local state of the vibration subsystem. The electron and pseudospin (lattice) contributions to the transverse dielectric susceptibility are investigated.
\end{abstract}

PACS numbers: 74.65.+n, 71.45.Gm

\section{Introduction}

A problem of high-temperature superconductivity (IITSC) is of major importance in contemporary solid state physics. Substantial attention has been recently paid to the study of theoretical models in view of the search for possible mechanisms of HTSC phenomena. These models are developed in the spirit of a Hubbard model, which describes strong electron correlations on a single site of a crystal lattice, and are supplemented by making allowance for peculiarities of either electron states or lattice dynamics for the crystals under consideration. Among these, one can consider the model for which the Hubbard Hamiltonian is supplemented by the interaction of electrons with a lattice vibration mode, which is characterized by essential local anharmonicity. The vibrations of apex oxygen $\left(\mathrm{O}_{\mathrm{IV}}\right)$ in the copper-oxide superconductors possess such a property [1]. In particular, the presence of two $\mathrm{Cu}-\mathrm{O}(4)$ distances follows from the analysis of EXAFS spectra for $\mathrm{YBaCuO}$ [2] manifesting the double potential well. This conclusion agrees with the experiments on the diffraction of X-rays [3]. An important role of 
the apex oxygen in the phase transition into superconductivity has been already mentioned $[4,5]$. The correlation between the oxygen energy spectrum and $T_{\mathrm{c}}$ was obtained [6]. The authors of Ref. [7] argue that the motion of $O(4)$ atoms normal to the $\mathrm{CuO}_{2}$ plane is characterized by strong fluctuations of charge along $c$-axis and electron (hole) transfer between these planes and chains of $\mathrm{CuO}$. It was shown in $[8,9]$ that the interaction of electrons and local anharmonic vibrations can lead to the superconductivity.

In addition, the coexistence of ITSC phenomena (in particular, in $\mathrm{YBaCuO}$ ) with ferro- or antiferroelectric ordering in the discussion of possible IITSC mechanism was questioned [10]. The role of instability of $\mathrm{O}^{2-}$ oxygen ion in this problem was noted by Bussman-IIolder, Simon and Buttner [11]. On this basis Müller et al. [12] have investigated the dielectric anomalies in the vicinity of $T_{\mathrm{c}}$ and possible instabilities due to violation of charge symmetry including the apex $\mathrm{O}_{\mathrm{IV}}$ atoms. It was shown that high values of dielectric susceptibility occur in $\mathrm{YBaCuO}$ compounds $[13,14]$. Besides, the symmetry loss with respect to the inversion at $T<T_{\mathrm{c}}$ was discussed. The data of piezoresistance and piezoelectricity measurements, observations of anomalies of ferro- or antiferroelectric type (see also [12]) prove the importance of the detailed study of dielectric properties of IITSC systems.

In order to describe anharmonic vibrations in case of the local potential with two minima separated by a high barrier, the pscudospin formalism can be applied (see [8,9]). Within this approximation the model [15], which allows for the interaction of electrons with local vibrations of ions (these are characterized by pseudospin variables $\left.\vec{S}_{i}, S_{i}^{z}= \pm 1 / 2\right)$ in the same cell

$$
H^{\prime}=\sum_{i} g\left(n_{i \uparrow}+n_{i \downarrow}\right) S_{i}^{z}
$$

was studied. Here $g n_{i}$ determines the change of electron state energy on the $i$-th site when oxygen transfers from the first to the second minimum. The total Hamiltonian of the model (it is also called the Müller model) contains the term $\Omega \sum_{i} S_{i}^{x}$, which describes tunneling decoupling of two lower vibrational levels of oxygen besides Eq. (1.1). The electron subsystem is given here by the IIubbard IIamiltonian. The similar Hamiltonian can be obtained in an excitonic mechanism [7]. It was also proposed [16] for the description of the propagation of the holes through a lattice of anions with filled shells.

This model has been mostly studied using the canonical transformation which allows one to exclude the interaction with local anharmonic vibrations providing the Hamiltonian with effective electron-electron interaction. The Cluster calculations [16] and Monte-Carlo simulations [17] have been also done.

A strong electron correlation on the single site leads to the splitting of electron spectrum into two subbands in the Iubbard model. Mutual influence of both subbands can be estimated within perturbation theory by the parameter $t / U(t$ is the transfer integral and $U$ is the energy of electron repulsion on the single site). Considering the lower subband, this leads to the effective IIamiltonian of the so-called $t-J$ model, where the influence of the upper subband appears as the exchange interelectron interaction $J_{i j}^{(0)}=-2 \ell_{i j}^{2} / U$, which is antiferromagnetic for $U>0$. The $t-J$ model was widely used recently for studies of $\mathrm{Cu}-\mathrm{O}$ layers elec- 
tron spectrum in HTSC and its peculiarities in the presence of antiferromagnetic exchange [18]. The transition into the ITSC state can also be described within the $t-J$ model [19].

In this paper our purpose is to study the spectrum of single-electron excitations at different electron concentrations and investigate the effective interelectron exchange interaction for the Müller model. Possible peculiarities of the electron exchange due to the presence and influence of the pseudospin subsystem (anharmonic lattice vibrations) will be considered. We shall allow for complicated character of single-electron spectrum, which differs from the IIubbard model by a larger number of subbands [20]. We have calculated transverse dielectric susceptibility on the basis of the Müller model applied for the system with two sublattices (containing, for instance, two CuO planes in the cell with opposite signs of asymmetry parameters for single-site potentials). The contribution into the static susceptibility from the electron and vibration (pseudospin) subsystem is included. The calculations were done in regimes with the constant electron concentration $(n=$ const) and constant value of chemical potential $(\mu=$ const), which provide a possibility to extract contributions due to the charge transfer effect from or to the plane.

\section{The Hamiltonian}

The Müller model [15] represents the anharmonic vibrations of the apex oxygen in an environment, where the electronic states of the $\mathrm{CuO}$-planes are described by the Hubbard model, which allows for the strong electron correlations at a single site. The Hamiltonian of the model has the following form:

$$
H=\sum_{i} H_{i}+\sum_{i, j, \sigma} t_{i j} a_{i \sigma}^{+} a_{j \sigma}
$$

where the single-site IIamiltonian $H_{i}$ describes the interaction of the CuO-plane electrons with the local anharmonic vibrations; $H_{i}$ is furthermore described in terms of pseudospin variables

$$
H_{i}=U n_{i \uparrow} n_{i \downarrow}+E_{0}\left(n_{i \uparrow}+n_{i \downarrow}\right)+g\left(n_{i \uparrow}+n_{i \downarrow}\right) S_{i}^{z}-\Omega S_{i}^{x}-h S_{i}^{z},
$$

$n_{i \sigma}=a_{i \sigma}^{+} a_{i \sigma}$ is the $\sigma$-spin electron number operator, $g n_{i}$ describes the change in energy of an $i$-site electron due to an oxygen transition from one minimum $\left(S^{z}=+1 / 2\right)$ of the anharmonic potential to another one $\left(S^{z}=-1 / 2\right)$. The magnitude of the $g$ parameter is of the order of the single-site interelectron Coulomb repulsion $U$ due to the strong oxygen polarizability of the CuO-planes. The last two terms in Eq. (2.2) describe the tunneling splitting of the vibrational mode and the asymmetry of the anharmonic potential. For the reasonable description of the physics of apex oxygen anharmonic vibrations, the case $\Omega<W$ (W is the halfwidth of a nonperturbed electron band) should be considered. For the purposes of the model analysis developed in the present paper, it is advisable to discuss different possible options (including $\Omega>W$ ) in order to clarify the feasibility of the model and the role of particular parameters. The case $\Omega>W$ was studied in Ref. [17], where the transition to a superconducting state was investigated with the help of Monte-Carlo simulation (possible numeric values of parameters are discussed 
there as well; see also $[16,21])$. We shall consider the wide range of asymmetry parameter $h$.

In the case of narrow bands $(t \ll U)$ and the absence of a pseudospin interaction, the single-site IIamiltonian (2.2) plays a role of a basic approximation. This suggests the following single-site basis of states $|R\rangle=\left|n_{i \uparrow}, n_{i \downarrow}, S_{i}^{z}\right\rangle$, which consists of eight state vectors
$|1\rangle=|0,0,1 / 2\rangle$,
$|\tilde{1}\rangle=|0,0,-1 / 2\rangle$,
$|2\rangle=|1,1,1 / 2\rangle$,
$|\tilde{1}\rangle=|1,1,-1 / 2\rangle$,
$|3\rangle=|0,1,1 / 2\rangle$,
$|\tilde{3}\rangle=|0,1,-1 / 2\rangle$,
$|4\rangle=|1,0,1 / 2\rangle$,
$|\tilde{4}\rangle=|1,0,-1 / 2\rangle$.

It is useful to introduce the Ilubbard operators $X^{R S}=|R\rangle\langle S|$ that act in the space spanned by the state vectors $(2.3)$

$$
\begin{aligned}
a_{i \uparrow} & =X_{i}^{14}+X_{i}^{32}+X_{i}^{\tilde{1} \tilde{4}}+X_{i}^{\tilde{3} \tilde{2}}, & a_{i \downarrow} & =X_{i}^{13}-X_{i}^{42}+X_{i}^{\tilde{1} \tilde{3}}-X_{i}^{\tilde{4} \tilde{2}}, \\
n_{i \uparrow} & =X_{i}^{44}+X_{i}^{22}+X_{i}^{\tilde{4} \tilde{4}}+X_{i}^{\tilde{2} \tilde{2}}, & n_{i \downarrow} & =X_{i}^{33}+X_{i}^{22}+X_{i}^{\tilde{3} \tilde{3}}+X_{i}^{\tilde{2} \tilde{2}}, \\
S_{i}^{z} & =\frac{1}{2} \sum_{R=1}^{4}\left(X_{i}^{R R}-X_{i}^{\widetilde{R} \tilde{R}}\right), & S_{i}^{x} & =\frac{1}{2} \sum_{R=1}^{4}\left(X_{i}^{R \widetilde{R}}+X_{i}^{\widetilde{R} R}\right) .
\end{aligned}
$$

Thus, we get the Hamiltonian $H_{i}$ in terms of the Ilubbard operators

$$
\begin{aligned}
H_{i}=\frac{h}{2} & \left(X_{i}^{\tilde{1} \tilde{1}}-X_{i}^{11}\right)+\left(E_{0}+\frac{g-h}{2}\right)\left(X_{i}^{33}+X_{i}^{44}\right) \\
& +\left(E_{0}-\frac{g-h}{2}\right)\left(X_{i}^{\tilde{3} \tilde{3}}+X_{i}^{\tilde{4} \tilde{4}}\right)+\left(2 E_{0}+U+\frac{2 g-h}{2}\right) X_{i}^{22} \\
& +\left(2 E_{0}+U-\frac{2 g-h}{2}\right) X_{i}^{\tilde{2} \tilde{2}}-\frac{\Omega}{2} \sum_{R=1}^{4}\left(X_{i}^{R \widetilde{R}}+X_{i}^{\widetilde{R} R}\right) .
\end{aligned}
$$

It is diagonal for $\Omega=0$ (i.e. no tunneling splitting of the vibrational states). For $\Omega \neq 0$ on the other hand, the transformation

with

$$
\left(\begin{array}{c}
|R\rangle \\
|\widetilde{R}\rangle
\end{array}\right)=\left\|\begin{array}{cc}
\cos \phi_{r} & \sin \phi_{r} \\
-\sin \phi_{r} & \cos \phi_{r}
\end{array}\right\|\left(\begin{array}{c}
|r\rangle \\
|\tilde{r}\rangle
\end{array}\right),
$$

$$
\cos 2 \phi_{r}=\frac{n_{r} g-h}{\sqrt{\left(n_{r} g-h\right)^{2}+\Omega^{2}}},
$$

where $n_{1}=0, n_{2}=2, n_{3}=n_{4}=1\left(n_{r}=n_{\bar{r}}\right)$, reduces (2.5) to a diagonal form and results in the IIamiltonian [20]

$$
H=\sum_{i, r} \varepsilon_{r} X_{i}^{r r}+\sum_{i, j, \sigma} t_{i j} a_{i \sigma}^{+} a_{j \sigma}
$$

with

$$
\begin{aligned}
& \varepsilon_{1, \tilde{1}}= \pm \frac{1}{2} \sqrt{h^{2}+\Omega^{2}}, \quad \varepsilon_{2, \tilde{2}}=2 E_{0}+U \pm \frac{1}{2} \sqrt{(2 g-h)^{2}+\Omega^{2}} \\
& \varepsilon_{3, \tilde{3}}=\varepsilon_{4, \tilde{4}}=E_{0} \pm \frac{1}{2} \sqrt{(g-h)^{2}+\Omega^{2}}
\end{aligned}
$$


and

$$
a_{i \sigma}^{+}=\sum_{m, n} A_{m n}^{\sigma} X_{i}^{m n}, \quad a_{i \sigma}=\sum_{m, n} A_{m n}^{\sigma} X_{i}^{n m},
$$

where $X^{m n}=|m\rangle\langle n|$ and

$$
\begin{array}{ll}
A_{41}^{\uparrow}=A_{\tilde{4} \tilde{1}}^{\uparrow}=\cos \left(\phi_{4}-\phi_{1}\right), & A_{23}^{\uparrow}=A_{\tilde{2} \tilde{3}}^{\uparrow}=\cos \left(\phi_{2}-\phi_{3}\right), \\
A_{\tilde{4} 1}^{\uparrow}=-A_{4 \tilde{1}}^{\uparrow}=\sin \left(\phi_{4}-\phi_{1}\right), & A_{\tilde{2} 3}^{\uparrow}=-A_{2 \tilde{3}}^{\uparrow}=\sin \left(\phi_{2}-\phi_{3}\right), \\
A_{31}^{\downarrow}=A_{\tilde{3} \tilde{1}}^{\downarrow}=\cos \left(\phi_{3}-\phi_{1}\right), & A_{24}^{\downarrow}=A_{\tilde{2} \tilde{4}}^{\downarrow}=-\cos \left(\phi_{2}-\phi_{4}\right), \\
A_{\tilde{3} 1}^{\downarrow}=-A_{3 \tilde{1}}^{\downarrow}=\sin \left(\phi_{3}-\phi_{1}\right), & A_{\tilde{2} 4}^{\downarrow}=-A_{2 \tilde{4}}^{\downarrow}=-\sin \left(\phi_{2}-\phi_{4}\right) .
\end{array}
$$

\section{Green's functions}

We calculate single-electron Green's functions from the equations of motion with the decoupling of high-order Grcen's functions in the spirit of the Hubbard-I approximation [22]

$$
\begin{aligned}
{\left[X_{i}^{n m}, H\right]=(} & \left.\varepsilon_{m}-\varepsilon_{n}\right) X_{i}^{n m}+\sum_{j, \sigma, p} t_{i j}\left[\left(A_{m p}^{\sigma} X_{i}^{n p}+A_{p n}^{\sigma} X_{i}^{p m}\right) a_{j \sigma}\right. \\
& \left.-a_{j \sigma}^{+}\left(A_{p m}^{\sigma} X_{i}^{n p}+A_{n p}^{\sigma} X_{i}^{p m}\right)\right] \approx\left(\varepsilon_{m}-\varepsilon_{n}\right) X_{i}^{n m} \\
& +\sum_{j, \sigma} t_{i j}\left(X_{i}^{n n}+X_{i}^{m m}\right\rangle\left(A_{m n}^{\sigma} a_{j \sigma}-A_{n m}^{\sigma} a_{j \sigma}^{+}\right) .
\end{aligned}
$$

After simple transformations we find Green's functions

$$
\left\langle\left\langle a_{\sigma} \mid X^{m n}\right\rangle\right\rangle_{q, \varepsilon}=\frac{1}{2 \pi} \frac{A_{m n}^{\sigma}}{1-t_{q} g_{\sigma}(\varepsilon)} \frac{\left\langle X^{m m}+X^{n n}\right\rangle}{\varepsilon-\left(\varepsilon_{m}-\varepsilon_{n}\right)}
$$

and

$$
\left\langle\left\langle a_{\sigma} \mid a_{\sigma}^{+}\right\rangle\right\rangle_{q, \varepsilon}=\frac{1}{2 \pi} \frac{1}{g_{\sigma}^{-1}(\varepsilon)-t_{q}},
$$

where $\iota_{q}$ is the Fourier transform of the transfer (hopping) term. The coupling to the apex oxygen is included by

$$
g_{\sigma}(\varepsilon)=\sum_{\nu} \frac{B_{\nu}}{\varepsilon-\varepsilon_{\nu}} \equiv \sum_{m, n} \frac{\left(A_{m n}^{\sigma}\right)^{2}\left(X^{m m}+X^{n n}\right\rangle}{\varepsilon-\left(\varepsilon_{m}-\varepsilon_{n}\right)}
$$

which is Green's function (3.3) for zero transfer. (The $\nu$-sum goes over all single-electron transitions.) The Hubbard-I approximation is available for narrow bands $\left(\left|t_{q}\right| \ll U\right.$ for the Hubbard model, or $\left|t_{q}\right| \ll\left|\varepsilon_{\nu}-\varepsilon_{\lambda}\right|(\nu \neq \lambda)$ for the considered model).

The spectrum of the single-electron transitions $\varepsilon_{\nu}\left(t_{q}\right)$ is, in turn, determined by zeros of Green's function (3.3) denominator. Equation (3.4) considers the homogeneous case, $\left(\left\langle X^{r r}\right\rangle\right.$ does not depend on the site number). We shall discuss the paramagnetic state $\left(n_{\downarrow}=n_{\uparrow}=n / 2,\left\langle X^{44}\right\rangle=\left\langle X^{33}\right\rangle,\left\langle X^{\tilde{4} \tilde{4}}\right\rangle=\left\langle X^{\tilde{3} \tilde{3}}\right\rangle\right.$ and 
$\left.g_{\uparrow}(\varepsilon)=g_{\downarrow}(\varepsilon)=g(\varepsilon)\right)$. The spectral theorem allows one to calculate the mean values

$$
\left\langle X^{m m}\right\rangle I_{m n}^{(-)}=\left\langle X^{n n}\right\rangle I_{m n}^{(+)}
$$

with

$$
I_{m n}^{( \pm)}=\frac{1}{\pi N} \sum_{q} \int_{-\infty}^{\infty} \frac{\mathrm{d} \varepsilon}{\mathrm{e}^{ \pm \beta(\varepsilon-\mu)}+1} \operatorname{Im}\left\{\frac{1}{\left[1-\operatorname{tg}_{q} g(\varepsilon)\right]\left[\varepsilon-\left(\varepsilon_{m}-\varepsilon_{n}\right)\right]}\right\},
$$

where $\mu$ is the chemical potential. The system of Eqs. (3.5) has to be completed by the normalization condition $\sum_{r} X^{r r}=1$ and by an equation for the chemical potential

$$
\langle\hat{n}\rangle=2\left\langle X^{22}+X^{33}+X^{\tilde{2} \tilde{2}}+X^{\tilde{3} \tilde{3}}\right\rangle=n .
$$

Further analytical calculations cannot be performed and we have to turn to numerical investigations. To do this, the $q$-summation in Eqs. (3.6) is changed to an integration over the rectangular density of states: $\rho(t)=1 /(2 W)$ for $-W \leq t \leq W$ and $\rho(t)=0$ otherwise. Equations from (3.4) to (3.7) define a self-consistent set, which allows one to calculate the spectrum of the single electron transitions and the mean values $\left\langle X^{r r}\right\rangle[20]$.

Only in case of the zero electron transfer $\left(t_{q}=0\right)$ the integrals (3.6) can be performed analytically and we get the Boltzmann distribution for the mean values $\left(E_{0}=-\mu\right)$

$$
\left\langle X^{r r}\right\rangle=\exp \left(-\beta \varepsilon_{r}\right) / \sum_{s} \exp \left(-\beta \varepsilon_{s}\right)
$$

An equation for the chemical potential $\mu$ results from a substitution of (3.8) into (3.7). A solution of this equation in the limit $\beta \rightarrow \infty$ shows that the result depends on the sign of an effective potential

$$
\begin{aligned}
U_{\mathrm{eff}}= & \left(\varepsilon_{\tilde{2}}-\varepsilon_{\tilde{3}}\right)-\left(\varepsilon_{\tilde{4}}-\varepsilon_{\tilde{1}}\right) \\
& =U-\left[\frac{1}{2} \sqrt{h^{2}+\Omega^{2}}+\frac{1}{2} \sqrt{(2 g-h)^{2}+\Omega^{2}}-\sqrt{(g-h)^{2}+\Omega^{2}}\right] .
\end{aligned}
$$

We get for positive values of $U_{\text {eff }}$ :

$$
\mu=\varepsilon_{\tilde{4}}-\varepsilon_{\tilde{1}}, \quad\left\langle X^{\tilde{1} \tilde{1}}\right\rangle=1-n, \quad\left\langle X^{\tilde{3} \tilde{3}}\right\rangle=\left\langle X^{\tilde{4} \tilde{4}}\right\rangle=\frac{n}{2}
$$

for $0<n<1$ and

$$
\mu=\varepsilon_{\tilde{2}}-\varepsilon_{\tilde{3}}, \quad\left\langle X^{\tilde{2} \tilde{2}}\right\rangle=n-1, \quad\left\langle X^{\tilde{3} \tilde{3}}\right\rangle=\left\langle X^{\tilde{4} \overline{4}}\right\rangle=1-\frac{n}{2}
$$

for $1<n<2$.

For negative values $U_{\text {eff }}$, on the other hand, we obtain

$$
\mu=\frac{1}{2}\left(\varepsilon_{\tilde{2}}-\varepsilon_{\tilde{1}}\right), \quad\left\langle X^{11}\right\rangle=1-\frac{n}{2}, \quad\left\langle X^{22}\right\rangle \frac{n}{2} .
$$

These solutions can also be obtained from the common IIubbard model for $t_{q}=0$ and for $U>0$ (repulsion) or $U<0$ (attraction), respectively. Thus, $U_{\text {eff }}$ is evidently the effective single-site electron-electron interaction of the electrons when an additional interaction of the electrons with the pseudospins is present (see [3]). 


\section{Independent subbands approximation}

In the first step we consider the case when the contribution of the transfer term is much smaller than the difference between two single-electron transition energies, i.e. $\left|t_{q}\right| \ll\left|\varepsilon_{\nu}-\varepsilon_{\lambda}\right|$ for $\nu \neq \lambda$. Starting from Eqs. (3.5) and (3.6) we obtain

and

$$
\varepsilon_{\nu}(t)=\varepsilon_{\nu}+t B_{\nu}
$$

$$
I_{\nu}^{( \pm)}=\frac{1}{2} \mp \frac{1}{2 \beta W B_{\nu}} \ln \frac{\operatorname{ch} \frac{\beta}{2}\left[\varepsilon_{\nu}(+W)-\mu\right]}{\operatorname{ch} \frac{\beta}{2}\left[\varepsilon_{\nu}(-W)-\mu\right]} .
$$

The single-electron transition levels

$$
\varepsilon_{\nu}=\varepsilon_{r s}=\varepsilon_{r}-\varepsilon_{s},
$$

with $\nu=(r, s)=\{(\tilde{4} 1),(\tilde{4} \tilde{1}),(41),(4 \tilde{1}),(\tilde{2} 3),(\tilde{2} \tilde{3}),(23),(2 \tilde{3})\}$ broaden into the bands (4.1) having a width of the order of $2 W B_{\nu}$.
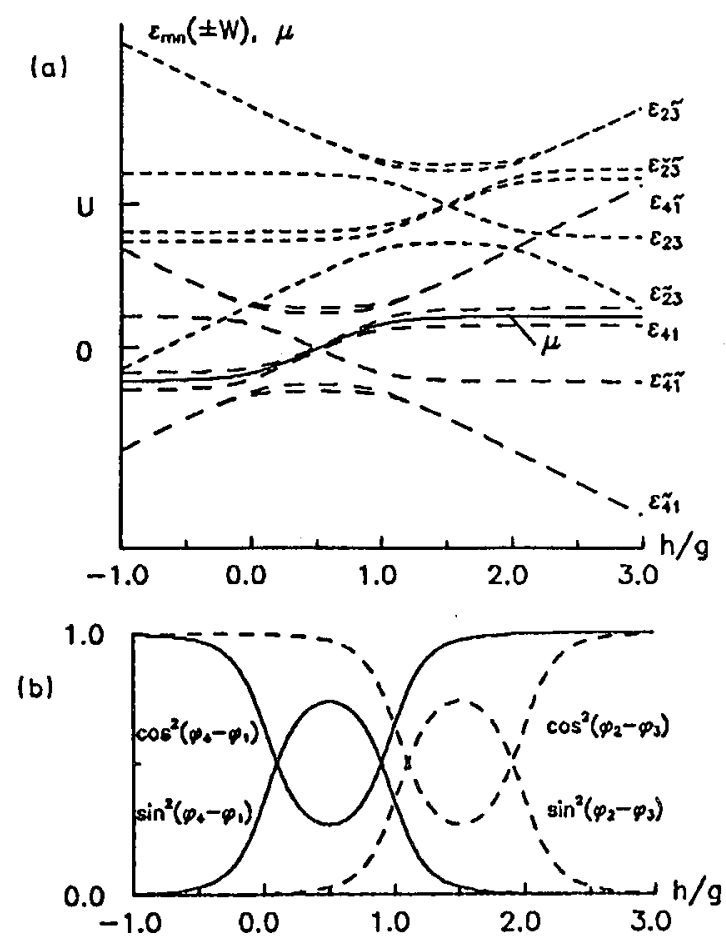

Fig. 1. The single-electron bands and chemical potential $\mu$ (a) and narrowing parameters $\left(A_{r s}^{\sigma}\right)^{2}(\mathrm{~b})$ as functions of the dimensionless coupling strength $h / g(U=2.2$, $g=1, \Omega=0.3, W=0.2, n=0.7)$.

Figure 1a presents the energies of the single-electron bands in the independent subbands approximation as functions of $h$. The interaction with the anharmonic (pseudospin) mode splits each energy level of the common IIubbard 
model ( $0, E_{0}$ and $\left.2 E_{0}+U\right)$ into two sublevels (3.9) (vibronic states) and, as the

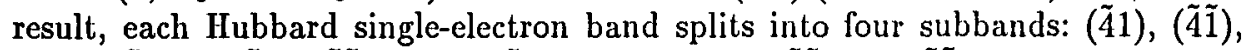

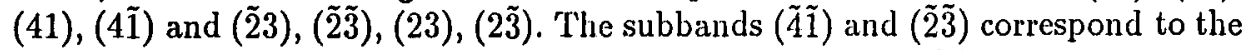
single-electron transitions between the lower vibronic states $|\tilde{r}\rangle$ and all the other include the excited vibronic states $|r\rangle$. The widths and statistical weights of the subbands are determined by the parameters $\left(A_{r s}^{\sigma}\right)^{2}=\left\{\cos ^{2}\left(\phi_{r}-\phi_{s}\right), \sin ^{2}\left(\phi_{r}-\phi_{s}\right)\right\}$ (Fig. 1b) and by the average occupancies of the states $\left\langle X^{m m}+X^{n n}\right\rangle$. Ilence, there are such values of $g$ and $h(0<h<g$ for $(\tilde{4} \tilde{1})$ and $g<h<2 g$ for $(\tilde{2} \tilde{3}))$ for which the subbands created by the lower vibronic states are strongly narrowed simultaneously with the broadening of the subbands formed by the excited states.
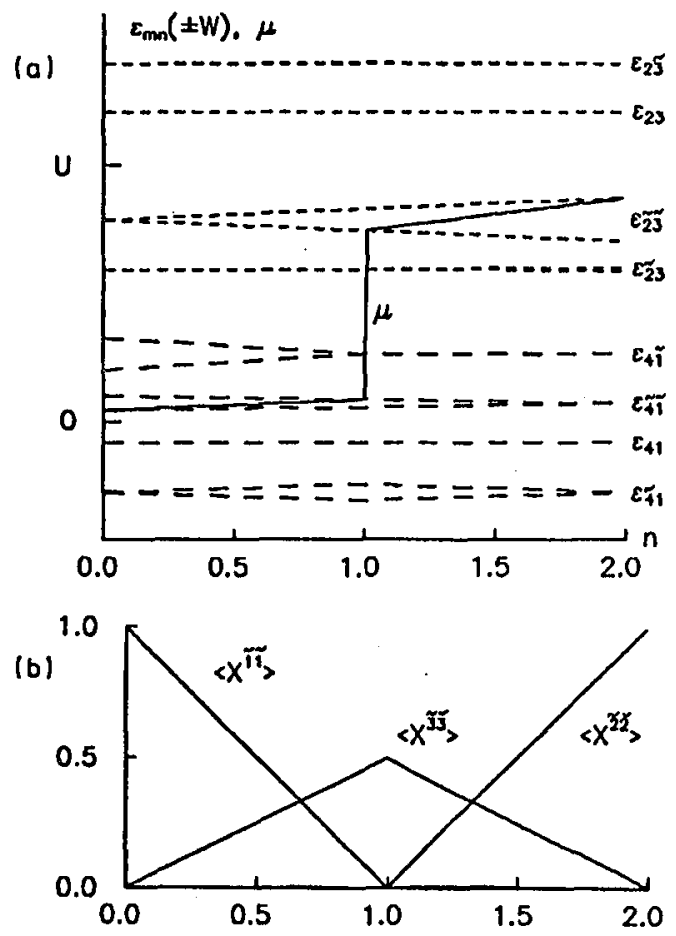

Fig. 2. The single-electron bands and chemical potential $\mu$ (a) and a verage occupancies $\left\langle X^{r r}\right\rangle$ (b) as functions of the electron concentration $n(U=2.2, g=1, h=0.7$, $\Omega=0.3, W=0.2, n=0.7)$.

Figure 2 shows how the spectrum, the chemical potential and the mean values of the Hubbard operators $\left\langle X^{r r}\right\rangle$ depend on the charge carrier concentration $n$. Obviously, the main effect comes from the $\tilde{1} \rightarrow \tilde{4}$ and $\tilde{3} \rightarrow \tilde{2}$ transitions with $\mu$ fixed within these bands.

The band interactions become important if the bands are not widely separated. This is observed for the following situations:

a) Small effective interactions $\left(U_{\text {eff }} \approx W\right)$ when the bands $(\tilde{4} \tilde{1})$ and $(\tilde{2} \tilde{3})$ 
become close or overlap;

b) Small tunneling frequencies $(\Omega<W)$ when the bands $(\tilde{4} \tilde{1})-(\tilde{4} 1),(\tilde{2} \tilde{3})-(2 \tilde{3})$ and $(\tilde{4} \tilde{1})-(4 \tilde{1}),(\tilde{2} \tilde{3})-(\tilde{2} 3)$ become near or overlap (see Fig. $1 \mathrm{a})$ for $h \approx 0,2 g$, and $g$, respectively. If the value of asymmetry parameter $h$ is far from these points we can neglect the interaction between such bands for all values of $\Omega$ (not only for $\Omega>W$ ); in these cases the band structure for $\Omega<W$ docs not differ radically from that for $\Omega>W$;

c) Effective attractions $\left(U_{\text {eff }}<0\right)$ when the chemical potential cannot be determined within independent subband approximation.

If the tunneling splitting $\Omega$ of the vibrational energy levels is less than the half of the electron band width $W$ and the asymmetry parameter $h$ is close to the above-mentioned values (case (b)), the chemical potential can also fix within $(r \tilde{s})$ or $(\tilde{r} s)$ bands (which were splitted off the $(\tilde{r} \tilde{s})$ band by the energy of the order of $\Omega$ ) for some values of the electron concentration. This case as well as the one of effective attraction (c) is discussed in details in [20].

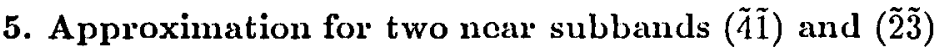

The ( $\tilde{4} \tilde{1})$ and $(\tilde{2} \tilde{3})$ subband interaction is treated taking the following terms in Eq. (3.4) for $g(\varepsilon)$ into account:

$$
g(\varepsilon)=\frac{B_{\overline{4} \overline{1}}}{\varepsilon-\varepsilon_{\overline{4} \tilde{1}}}+\frac{B_{\overline{2} \tilde{3}}}{\varepsilon-\varepsilon_{\tilde{2} \tilde{3}}},
$$

with

$$
B_{\tilde{1} \tilde{4}}=\cos ^{2}\left(\phi_{4}-\phi_{1}\right)\left\langle X^{\overline{1} \tilde{1}}+X^{\tilde{4} \tilde{4}}\right\rangle, \quad B_{\tilde{2} \tilde{3}}=\cos ^{2}\left(\phi_{2}-\phi_{3}\right)\left(X^{\tilde{3} \tilde{3}}+X^{\tilde{2} \tilde{2}}\right\rangle .
$$

There are no couplings with the subbands formed by the excited states for $\left|\varepsilon_{\nu}-\varepsilon_{\lambda}\right|>W, 1 / \beta(\nu \neq \lambda)$ and we can put

$$
\left\langle X^{\overline{1} \tilde{1}}+X^{\tilde{4} \tilde{4}}\right\rangle=1-\frac{n}{2}, \quad\left\langle X^{\tilde{3} \tilde{3}}+X^{\tilde{2} \overline{2}}\right\rangle=\frac{n}{2} .
$$

Solving (3.5) results in the spectrum

$$
\begin{aligned}
\varepsilon_{\mathrm{I}, \mathrm{II}}(t)= & a(t) \pm b(t) \equiv \frac{1}{2}\left(\varepsilon_{\tilde{4} \tilde{1}}+t B_{\tilde{4} \tilde{1}}+\varepsilon_{\tilde{2} \tilde{3}}+t B_{\tilde{2} \tilde{3}}\right) \\
& \pm \frac{1}{2} \sqrt{\left(\varepsilon_{\tilde{3} \tilde{1}}+t B_{\overline{4} \tilde{1}}-\varepsilon_{\overline{2} \tilde{3}}-t B_{\overline{2} \overline{3}}\right)^{2}+4 t^{2} B_{\overline{4} \overline{1}} B_{\overline{2} \tilde{3}}} .
\end{aligned}
$$

We can also calculate the integrals (3.6) which give now

$$
\begin{aligned}
& I_{\tilde{4} \tilde{1}}^{( \pm)}(\mu)=\frac{1}{2} \mp I_{1}(\mu) \pm B_{\tilde{2} \overline{3}} I_{2}(\mu), \\
& I_{\tilde{2} \tilde{3}}^{( \pm)}(\mu)=\frac{1}{2} \mp I_{1}(\mu) \mp B_{\tilde{4} \tilde{1}} I_{2}(\mu),
\end{aligned}
$$

with

$$
\begin{aligned}
& I_{1}(\mu)=\frac{1}{2 \beta W\left(B_{\tilde{4} \tilde{1}}+B_{\tilde{2} \tilde{3}}\right)} \ln \frac{\operatorname{ch} \beta[a(+W)-\mu]+\operatorname{ch} \beta b(+W)}{\operatorname{ch} \beta[a(-W)-\mu]+\operatorname{ch} \beta b(-W)} \\
& I_{2}(\mu)=\frac{1}{4 W\left(B_{\tilde{4} \tilde{1}}+B_{\tilde{2} \tilde{3}}\right)} \int_{-W}^{W} \frac{\mathrm{d} t}{b(t)} \frac{\operatorname{sh} \beta b(t)}{\operatorname{ch} \beta[a(t)-\mu]+\operatorname{ch} \beta b(t)}
\end{aligned}
$$


where we set $E_{0}=0$. The other subbands are treated in the independent subbands approximation.

Such an approximation is similar to that which is usually used for the IIubbard model with an effective Coulomb repulsion (3.9) and with the hopping integrals

$$
t_{\overline{4} \overline{1}}=t \cos ^{2}\left(\phi_{4}-\phi_{1}\right), \quad t_{\overline{2} \overline{3}}=t \cos ^{2}\left(\phi_{2}-\phi_{3}\right),
$$

which results from the bands "narrowing" due to the interaction with an anharmonic mode (see c.f. [3]).

We substitute (5.2) into (5.6) and calculate the mean values of the Hubbard operators. The equation for chemical potential has the form

$$
I_{1}(\mu)-\frac{1-n}{2}=I_{2}(\mu) \frac{n}{2}\left(1-\frac{n}{2}\right)\left[\cos ^{2}\left(\phi_{2}-\phi_{3}\right)-\cos ^{2}\left(\phi_{4}-\phi_{1}\right)\right] .
$$

Our results differ from the Ilubbard model mainly in the factors of $\cos ^{2}\left(\phi_{4}-\phi_{1}\right)$ and $\cos ^{2}\left(\phi_{2}-\phi_{3}\right)$ which characterize the narrowing of the subbands. In the limit $g=0$ (i.e. no interaction with the pseudospin) $\cos ^{2}\left(\varphi_{4}-\varphi_{1}\right)=$ $\cos ^{2}\left(\phi_{2}-\phi_{3}\right)=1$ and $B_{\overline{4} \overline{1}}+B_{\overline{2} \overline{3}}=1$ which makes the right hand side of Eq. (5.8) equal to zero.

The results for positive values of $U_{\text {eft }}$ are qualitatively identical to those of the Hubbard model. The only difference consists of the jump of the chemical potential from the lower to the upper band which occurs now for $n_{0} \neq 1$. Therefore, at half-filling $(n=1)$ we have the hole conductivity for $\cos ^{2}\left(\phi_{4}-\phi_{1}\right)<\cos ^{2}\left(\phi_{2}-\phi_{3}\right)$ and electron conductivity on the opposite which comes from the effective "band narrowing" in the presence of the interaction with anharmonic modes. For small band widths ( $\left.W \ll U_{\text {eff }}\right) n_{0}$ is determined from

$$
n_{0}=1+\frac{1}{6}\left(\frac{W}{U_{\text {eff }}}\right)^{2}\left[\cos ^{4}\left(\phi_{2}-\phi_{3}\right)-\cos ^{4}\left(\phi_{4}-\phi_{1}\right)\right]+\ldots
$$

and we find out that the interaction with the pseudospin destroys the electron-hole symmetry.

\section{Effective exchange interaction}

In the Iubbard model the interband coupling can be considered using the perturbation theory in parameter $t / U$. For lower Irubbard band it leads to the effective Hamiltonian of the so-called $t-J$ model, where the influence of the upper band manifests itself as the effective electron-electron exchange interaction $J_{i j}^{\text {eff }}=$ $-2 t_{i j}^{2} / U$, which is antiferromagnetic for $U>0$.

The hopping term of the Hamiltonian (2.1) can be rewritten in the form

$$
H_{\mathrm{d}}+H^{\prime} \equiv \sum_{i, j} t_{i j} \sum_{m, n} a_{m n} X_{i}^{m n} X_{j}^{n m}+\sum_{i, j} t_{i j} \sum_{m, n, m^{\prime}, n^{\prime}}^{\prime} b_{m n, m^{\prime} n^{\prime}} X_{i}^{m n} X_{j}^{n^{\prime} m^{\prime}} \text {, }
$$

where

$$
a_{m n}=\sum_{\sigma}\left(A_{m n}^{\sigma}\right)^{2}, \quad b_{m n, m^{\prime} n^{\prime}}=\sum_{\sigma} A_{m n}^{\sigma} A_{m^{\prime} n^{\prime}}^{\sigma}
$$


Now the total Hamiltonian has the form

$$
H=\left(H_{0}+H_{\mathrm{d}}\right)+H^{\prime}, \quad H_{0}=\sum_{i, r} \varepsilon_{r} X_{i}^{r r} .
$$

Similarly to the standard transition from the IIubbard model to the $t-J$ one, we perform the canonical transformation in the form [23-25]

$$
H \rightarrow \bar{H}=\mathrm{e}^{-\mathrm{i} \widehat{S}} H \mathrm{e}^{\mathrm{i} \widehat{S}},
$$

where the generator $\widehat{S}=\widehat{S}^{+}$is determined from the condition

$$
\widehat{H}^{\prime}+\mathrm{i}\left[\widehat{S}, \widehat{H}_{0}\right]=0 \text {. }
$$

As the result, the total Ilamiltonian (6.3) with accuracy up to the second order in $H^{\prime}$ has the form

$$
H=\left(H_{0}+H_{d}\right)+\mathrm{i}\left[\widehat{S}, \hat{H}_{d}\right]+\frac{\mathrm{i}}{2}\left[\widehat{S}, \hat{H}^{\prime}\right] .
$$

We look for the generator $\widehat{S}$ in the form

$$
\mathrm{i} \widehat{S}=\sum_{i, j} \sum_{p, q, r, s}^{\prime} C_{i j}^{p q, r s} X_{i}^{p q} X_{j}^{s r} .
$$

From a substitution of (6.7) into (6.5) we find

$$
C_{i j}^{p q, r s}=-\frac{t_{i j} b_{p q, r s}}{\varepsilon_{q}-\varepsilon_{p}+\varepsilon_{r}-\varepsilon_{s}} .
$$

It is clear that such a perturbation theory is only correct when the hopping integral is much smaller than the distance between the single-electron transition levels $\left(\left|t_{i j}\right| \ll\left|\varepsilon_{p q}-\varepsilon_{r s}\right|\right)$. By substitution of (6.7) with (6.8) into (6.6) we obtain the expression which contains three-site indices and there are the cases when two of them can coincide. Taking into account such terms we find

$$
\begin{aligned}
\frac{\mathrm{i}}{2}\left[\widehat{S}, \hat{H}^{\prime}\right]= & \sum_{i, j} t_{i j}^{2} \sum_{p, q, r, s}^{\prime} \sum_{q_{1}, r_{1}}^{\prime} b_{p q, r_{s}} b_{q_{1}, r_{1}} \\
& \times\left[\frac{1}{\varepsilon_{q}-\varepsilon_{p}+\varepsilon_{r}-\varepsilon_{s}}+\frac{1}{\varepsilon_{q_{1}}-\varepsilon_{q}+\varepsilon_{r_{1}}-\varepsilon_{r}}\right] X_{i}^{p q_{1}} X_{j i}^{s r_{1}} .
\end{aligned}
$$

This expression describes the effective interaction in the second order in $t$. In Eq. (6.9) there are terms corresponding to the effective exchange. In the approximation resulting in the IIamiltonian of the $t-J$ model, these terms become the leading ones.

Introducing the pseudospin operators $S^{z}, S^{+}$and $S^{-}$similar to (2.4), but acting in the space spanned by the eigenstates of the single-site Ilamiltonian (2.2), the exchange part of (6.9) can be written in the form [26]

$$
\begin{aligned}
& -\sum_{i, j}\left\{J_{i j}^{++} P_{i}^{+} P_{j}^{+}+J_{i j}^{--} P_{i}^{-} P_{j}^{-}+J_{i j}^{+-}\left(P_{i}^{+} P_{j}^{-}+P_{i}^{-} P_{j}^{+}\right)\right. \\
& +\left(J_{i j}^{\uparrow \uparrow}+J_{i j}^{\lfloor\downarrow}\right)\left(S_{i}^{+} S_{j}^{+}+S_{i}^{-} S_{j}^{-}\right)+J_{i j}^{\dagger \downarrow}\left(S_{i}^{+} S_{j}^{-}+S_{i}^{-} S_{j}^{+}\right) \\
& +\frac{1}{2}\left(J_{i j}^{+\uparrow}+J_{i j}^{\downarrow+}\right)\left[P_{i}^{+}\left(S_{j}^{+}+S_{j}^{-}\right)+P_{j}^{+}\left(S_{i}^{+}+S_{i}^{-}\right)\right]
\end{aligned}
$$




$$
\begin{aligned}
& \left.+\frac{1}{2}\left(J_{i j}^{-\dagger}+J_{i j}^{\downarrow-}\right)\left[P_{i}^{-}\left(S_{j}^{+}+S_{j}^{-}\right)+P_{j}^{-}\left(S_{i}^{+}+S_{i}^{-}\right)\right]\right\} \\
& \times\left(\vec{\sigma}_{i} \vec{\sigma}_{j}-\frac{1}{4} n_{i} n_{j}\right),
\end{aligned}
$$

where $P_{i}^{ \pm}=(1 / 2) \pm S_{i}^{z}, S_{i}^{z}=(1 / 2) \sum_{p=1}^{4}\left(X_{i}^{p p}-X_{i}^{\tilde{p} \tilde{p}}\right), S_{i}^{+}=\sum_{p=1}^{4} X_{i}^{p \tilde{p}}, S_{i}^{-}=$ $\sum_{p=1}^{4} X_{i}^{\tilde{p} p}$ and $\vec{\sigma}_{i}$ is the electron spin operator.

The expression in braces is the effective electron-electron exchange integral for the Müller model. This integral now depends on pseudospin operators, in contrast to the $t-J$ model case where the eflective exchange is constant $J_{i j}^{(0)}=-2 t_{i j}^{2} / U$. The values and the signs of the coeflicients $J_{i j}^{\alpha \beta}$ are determined by the denominators $\varepsilon_{p q}-\varepsilon_{r s}$ and, in particular, by the effective single-site electron-electron interaction $U_{\text {eff }}(3.9)$

$$
\begin{aligned}
& J_{i j}^{++}=-t_{i j}^{2} \sum_{p, q} \frac{\left(A_{4 p}^{\uparrow}\right)^{2}\left(A_{q 3}^{\uparrow}\right)^{2}+\left(A_{3 p}^{\downarrow}\right)^{2}\left(A_{q 4}^{\downarrow}\right)^{2}}{\varepsilon_{p}+\varepsilon_{q}-\varepsilon_{3}-\varepsilon_{4}} \\
& J_{i j}^{--}=-t_{i j}^{2} \sum_{p, q} \frac{\left(A_{\overline{4} p}^{\dagger}\right)^{2}\left(A_{q \overline{3}}^{\dagger}\right)^{2}+\left(A_{\tilde{3} p}^{\downarrow}\right)^{2}\left(A_{q \bar{q}}^{\downarrow}\right)^{2}}{\varepsilon_{p}+\varepsilon_{q}-\varepsilon_{\tilde{3}}-\varepsilon_{\overline{4}}}, \\
& J_{i j}^{+-}=-t_{i j}^{2} \sum_{p, q} \frac{\left(A_{\overline{4} p}^{\uparrow}\right)^{2}\left(A_{q 3}^{\dagger}\right)^{2}+\left(A_{3 p}^{\downarrow}\right)^{2}\left(A_{q \bar{q}}^{\downarrow}\right)^{2}}{\varepsilon_{p}+\varepsilon_{q}-\varepsilon_{3}-\varepsilon_{\overline{4}}}, \\
& J_{i j}^{\uparrow \uparrow}=\sum_{p, q} \frac{t_{i j}^{2} S_{p q}}{\varepsilon_{p}+\varepsilon_{q}-\varepsilon_{3}-\varepsilon_{4}}, \quad J_{i j}^{\downarrow \downarrow}=\sum_{p, q} \frac{t_{i j}^{2} S_{p q}}{\varepsilon_{p}+\varepsilon_{q}-\varepsilon_{\tilde{3}}-\varepsilon_{\overline{4}}}, \\
& J_{i j}^{\uparrow \downarrow}=\sum_{p, q} \frac{t_{i j}^{2} S_{p q}}{\varepsilon_{p}+\varepsilon_{q}-\varepsilon_{3}-\varepsilon_{\dot{q}}}, \\
& J_{i j}^{+\uparrow}=t_{i j}^{2} \sum_{p, q} \frac{\left(A_{4 p}^{\uparrow}\right)^{2} A_{q 3}^{\dagger} A_{q \tilde{3}}^{\dagger}+A_{\tilde{3} p}^{\downarrow} A_{3 p}^{\downarrow}\left(A_{q 4}^{\downarrow}\right)^{2}}{\varepsilon_{p}+\varepsilon_{q}-\varepsilon_{3}-\varepsilon_{4}}, \\
& J_{i j}^{-\uparrow}=t_{i j}^{2} \sum_{p, q} \frac{\left(A_{\dot{4} p}^{\dagger}\right)^{2} A_{q 3}^{\dagger} A_{q \overline{3}}^{\dagger}+A_{\overline{3} p}^{\perp} A_{3 p}^{\downarrow}\left(A_{q \bar{q}}^{\downarrow}\right)^{2}}{\varepsilon_{p}+\varepsilon_{q}-\varepsilon_{3}-\varepsilon_{\overline{4}}}, \\
& J_{i j}^{\downarrow+}=t_{i j}^{2} \sum_{p, q} \frac{A_{\overline{4} p}^{\uparrow} A_{4 p}^{\uparrow}\left(A_{q 3}^{\uparrow}\right)^{2}+\left(A_{3 p}^{\downarrow}\right)^{2} A_{q 4}^{\downarrow} A_{q \overline{4}}^{\downarrow}}{\varepsilon_{p}+\varepsilon_{q}-\varepsilon_{3}-\varepsilon_{\tilde{4}}},
\end{aligned}
$$




$$
J_{i j}^{\downarrow-}=t_{i j}^{2} \sum_{p, q} \frac{A_{\overline{4} p}^{\uparrow} A_{4 p}^{\uparrow}\left(A_{q \tilde{3}}^{\uparrow}\right)^{2}+\left(A_{\tilde{3} p}^{\downarrow}\right)^{2} A_{q 4}^{\downarrow} A_{q \overline{4}}^{\downarrow}}{\varepsilon_{p}+\varepsilon_{q}-\varepsilon_{\tilde{3}}-\varepsilon_{\tilde{4}}},
$$

where $S_{p q}=A_{\tilde{4} p}^{\uparrow} A_{4 p}^{\uparrow} A_{q 3}^{\uparrow} A_{q \tilde{3}}^{\uparrow}+A_{\tilde{3} p}^{\downarrow} A_{3 p}^{\downarrow} A_{q 4}^{\downarrow} A_{q \overline{4}}^{\downarrow}$. Simpler expressions are obtained for $\Omega=0$ when

$$
J_{i j}^{++}=J_{i j}^{--}=-\frac{2 t_{i j}^{2}}{U}, \quad J_{i j}^{+-}=-\frac{2 t_{i j}^{2} U}{U^{2}-g^{2}}
$$

and all other are equal to zero.

The total effective exchange integral contains components $J_{i j}^{ \pm \pm}, J_{i j}^{--}$, which describe the exchange interaction when the pseudospins in neighbouring sites are in fixed states $\left(S_{i}^{z}=1 / 2\right.$ or $\left.-1 / 2\right)$ and components $J_{i j}^{\uparrow \uparrow}, J_{i j}^{\uparrow \uparrow}, J_{i j}^{+\uparrow}$, etc. which describe more complicated effects when the electron exchange is accompanied by the pseudospin flipping (for $\Omega \neq 0$ ).
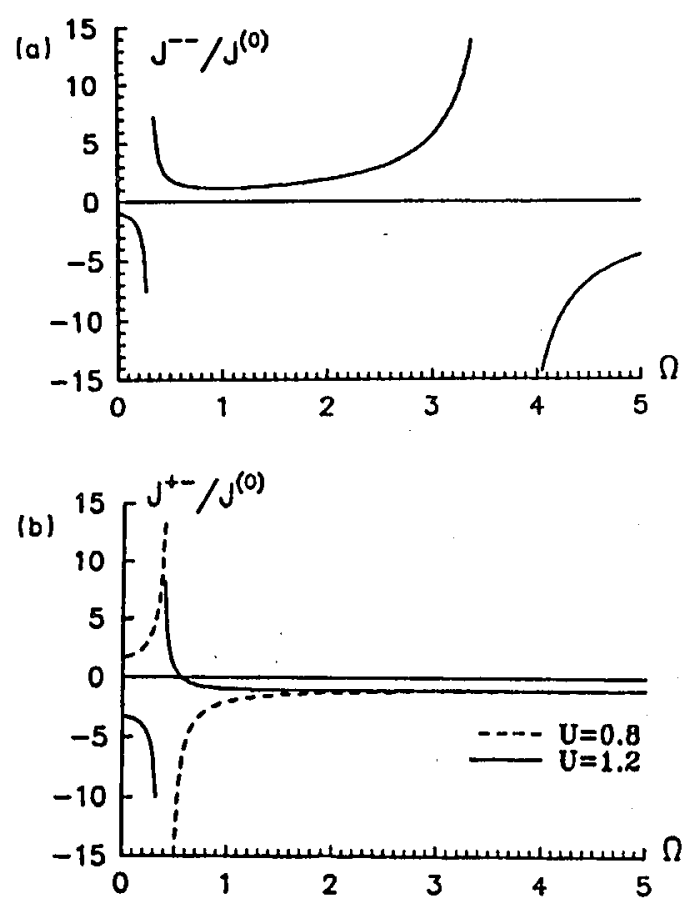

Fig. 3. The dependence of the $J^{--}(U=0.12)$ (a) and $J^{+-}$(b) effective exchange components on $\Omega\left(g=1, h=0, J_{i j}^{(0)}=2 t_{i j}^{2} / U\right)$.

Figure 3ab presents exchange constants $J_{i j}^{--}$and $J_{i j}^{+-}$defined by the formulas (6.11) vs. tunneling energy $\Omega$. The exchange constants diverge and change the sign when the values of corresponding denominators in (6.11) (U $U_{\text {eft }}=\varepsilon_{\tilde{2}}+\varepsilon_{\tilde{1}}-\varepsilon_{\tilde{\mathbf{3}}}-\varepsilon_{\tilde{4}}$ for $J_{i j}^{--}$and $\varepsilon_{\tilde{2}}+\varepsilon_{1, \tilde{1}}-\varepsilon_{\tilde{3}}-\varepsilon_{\overline{4}}$ for $J_{i j}^{+-}$) cross zero value. Nevertheless, as it was 
mentioned above, the perturbation theory expansion (6.6) and, next, the expressions (6.10)-(6.12) are correct only when $\left|t_{i j}\right| \ll\left|\varepsilon_{p q}-\varepsilon_{r s}\right|$ and cannot apply when the energy denominators are smaller than the hopping term $t_{i j}$.

In Fig. 3a the regions with the antiferromagnetic exchange interaction $J_{i j}^{--}<0$ correspond to the positive values of the eflective interaction $U_{\text {eff }}>0$. In the intermediate region $U_{\text {eff }}<0$ and the effective exchange is ferromagnetic, $J_{i j}^{--}>0$, but it does not lead to the ferromagnetic ordering because in this case the homopolarity is broken, electrons couple into pairs (see Eq. (3.12)) and $\left\langle\sigma_{i}^{z}\right\rangle=\left(n_{i \uparrow}-n_{i \downarrow}\right) / 2 \rightarrow 0$.

On the other hand, the value and the sign of the exchange constant $J_{i j}^{+-}$ are not determined by $U_{\text {eff }}$ but by the denominators $\varepsilon_{\tilde{2}}+\varepsilon_{1, \tilde{1}}-\varepsilon_{\tilde{3}}-\varepsilon_{\tilde{4}}$ and the exchange interaction between the sites with opposite directions of pseudospins can possess either antiferromagnetic or ferromagnetic character due to the sign of the denominator.

The total exchange interaction is determined by the state of the pseudospin subsystem. If the crystal is in the equilibrium state, then at low temperatures $\left\langle S_{i}^{z}\right\rangle=(-1 / 2)\left(\left\langle P^{+}\right\rangle=0,\left\langle P^{-}\right\rangle=1\right)$ and $J_{i j}^{-}$produces the main contribution to the total exchange.

When vibrational subsystem is nonequilibrium (e.g. in frozen disorder state, which can be realized in IITSC materials with nonstoichiometry in oxygen) the number of sites with the $S_{i}^{z}=+1 / 2$ values of the pseudospins increases and $J_{i j}^{+-}$and $J_{i j}^{++}$give the contribution to the total exchange. Now, the exchange interaction between the different sites has different values and signs depending on the distribution of the pseudospins in the lattice. This situation can correspond to the frustration of the exchange interaction discussed in some papers [27, 28].

\section{Dielectric properties of the model}

In order to investigate dielectric properties of the system which is described by the Müller model, let us introduce into the IIamiltonian the interaction energy with the external field $E_{\perp}$

$$
H_{\mathrm{int}}=-E_{\perp} \sum_{i} \Delta P_{i}^{z},
$$

where $\Delta P_{i}^{z}$ is the variable part of the transverse (i.e. normal to the $\mathrm{CuO}$ layer) component of the polarization vector

$$
\Delta P_{i}^{z}=\left(d_{\mathrm{s}} S_{i}^{z}+d_{\mathrm{e}} \widehat{n}_{i}\right) .
$$

Here $d_{\mathrm{s}}$ is the dipole moment connected with the pseudospin turning (transition of the oxygen atom from one minimum of the double well potential to the other one); $d_{\mathrm{e}}$ is the dipole moment corresponding to the electron charge transfer from (or to) the layer of $\mathrm{CuO}$ ). We do not include into the IIamiltonian the states to which the electron transitions from the layers of $\mathrm{CuO}$ are possible (these are, first of all, electron levels of the $\mathrm{CuO}$ chains). It is supposed that in case of the thermodynamic equilibrium there exists a reservoir of electrons which determines the chemical potential level in the layer. 
Calculating the dielectric susceptibility

$$
\chi=\frac{1}{v_{\mathrm{c}}} \frac{\partial P^{z}}{\partial E_{\perp}}
$$

where $v_{c}$ is the volume of a primitive cell, we will consider both situations, when $\mu=$ const and when $n=$ const. It will give us the possibility to investigate the polarization in case of absence of the electron charge transfer in the normal direction to the layer.

This problem can be easily generalized to the case of the crystal with two sublattices (two layers of $\mathrm{CuO}$ in the primitive cell, as in the $\mathrm{YBaCuO}$ crystal). Because of the fact that the lattice is centrosymmetric, the layers differ in the signs of the dipole moment

$$
d_{\mathrm{s}}^{(1)}=-d_{\mathrm{s}}^{(2)}, \quad d_{\mathrm{e}}^{(1)}=-d_{\mathrm{e}}^{(2)} .
$$

That is why the contributions from the layers are mutually compensated in the total spontaneous polarization $\left(\Delta P_{i(1)}^{z}+\Delta P_{i(2)}^{z}=0\right)$ and are summed in the susceptibility

$$
\chi_{\perp}^{\text {total }}=\frac{2}{v_{c}} \frac{\partial\left\langle P_{i(1)}^{z}\right\rangle}{\partial E_{\perp}}
$$

If one does not take into account the interaction between diflerent layers, it is possible to consider the problem about transverse polarization of one layer. In this case

$$
x_{\perp}^{\text {total }}=\frac{2}{v_{\mathrm{c}}}\left[d_{\mathrm{s}}\left(\frac{\partial\left\langle S_{i}^{z}\right\rangle}{\partial E_{\perp}}\right)_{\mu}+d_{\mathrm{e}}\left(\frac{\partial\left\langle n_{i}\right\rangle}{\partial E_{\perp}}\right)_{\mu}\right]
$$

for $\mu=$ const, and

$$
\chi_{\perp}^{\text {total }}=\frac{2}{v_{\mathrm{c}}} d_{\mathrm{s}}\left(\frac{\partial\left\langle S_{i}^{z}\right\rangle}{\partial E_{\perp}}\right)_{n}
$$

for $n=$ const.

We find the average values of $\left\langle S_{i}^{z}\right\rangle$ and $\left\langle n_{i}\right\rangle$ in the presence of the field $E_{\perp}$ in the approximation, which corresponds to that applied in Secs. 2-6 with the help of the following relations

$$
\left\langle S_{i}^{z}\right\rangle=\frac{1}{2} \sum_{r=1}^{4} \cos 2 \varphi_{r}\left\langle X_{i}^{r r}-X_{i}^{\tilde{r} \tilde{r}}\right\rangle, \quad\left\langle n_{i}\right\rangle=\sum_{r=1}^{4} n_{r}\left\langle X_{i}^{r r}+X_{i}^{\tilde{r} \tilde{r}}\right\rangle
$$

The results obtained there can be directly used if one takes into account the fact that the consideration of interaction with the external field leads formally to the substitutions of variables

$$
E_{0} \rightarrow E_{0}-d_{\mathrm{e}} E_{\perp}, \quad h \rightarrow h+d_{\mathrm{s}} E_{\perp} .
$$

The mean values $\left\langle S_{i}^{z}\right\rangle$ and $\left\langle n_{i}\right\rangle$ as functions of $E_{\perp}$ as well as the derivations $\left(\frac{\partial\left\langle S_{i}^{*}\right\rangle}{\partial E_{\perp}}\right)_{\mu},\left(\frac{\partial\left\langle S_{i}^{*}\right\rangle}{\partial E_{\perp}}\right)_{n}$ and $\left(\frac{\partial\left\langle n_{i}\right\rangle}{\partial E_{\perp}}\right)_{\mu}$ were obtained by means of numerical calculations. 
In case of $n=$ const the average occupancies of states $\left\langle X^{r r}\right\rangle$ weakly depend on the field and the main contribution into susceptibility gives the pseudospin subsystem, then

$$
\chi_{\perp}^{\text {total }}=\frac{d_{\mathrm{s}}^{2}}{v_{\mathrm{c}}} \sum_{r=1}^{4} \frac{\Omega^{2}}{\left[\left(n_{r} g-h\right)^{2}+\Omega^{2}\right]^{3 / 2}}\left\langle X^{\bar{r} \tilde{r}}-X^{r r r}\right\rangle
$$

The expression (7.10) contains three terms $\left(n_{r}=0,2\right.$ and 1), each of them describing the susceptibility of a single spin is placed in the transverse field $\Omega$ and longitudinal field $\left(h-n_{r} g\right)$. The possible peak of susceptibility at $h=n_{r} g$ corresponds to each of these terms; the weiglit of each peak is determined by the values $\left\langle X^{\tilde{r} \tilde{r}}-X^{r r}\right\rangle$. Figure 4a illustrates situations which take place in regimes $n=1$
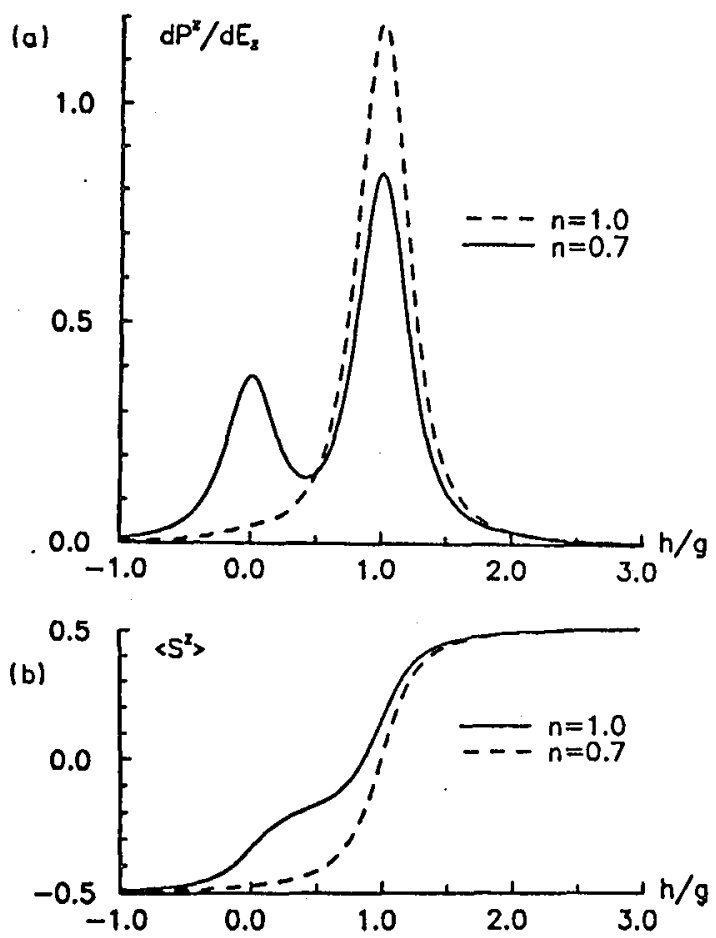

Fig. 4. The dielectric susceptibility (a) and pseudospin mean value (b) vs. $h / g$ for different electron concentrations $\left(U=2.2, g=1, h=0.7, \Omega=0.3, W=0.2, d_{s}=0.6\right)$; here and below susceptibility $\chi=\left(1 / v_{\mathrm{c}}\right) \partial P^{z} / \partial E_{\perp}$ is given in $d_{\mathrm{c}}^{2} / v_{\mathrm{c}} g$ units.

and $n<1$. There is one peak for $n=1$ at $h=g$; witl a decrease in $n$ a peak at $h=0$ appears and increases, whereas the intensity of the first peak decreases. Positions of the peaks correlate with the behaviour of the parameter $\left\langle S^{z}\right\rangle$ with a change of $h$ (Fig. 4b) and correspond to the values of asymmetry parameter for which the character of occupancies of double-well minima changes. 
Whereas at $n=$ const the chemical potential is always in one of the subbands (or between them), in the regime $\mu=$ const it becomes possible that the chemical potential goes out from (or enters into) the permitted energies band.

It follows from the above calculations that when the chemical potential is outside the bands $(\tilde{4} \tilde{1})$ and $(\tilde{2} \tilde{3})$, the concentration $n$ of electrons in layer is almost

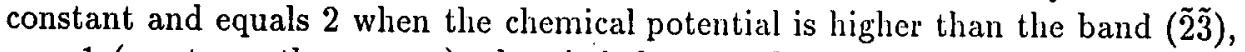
$n=1$ (most exactly $n=n_{0}$ ) when it is between bands and $n=0$ if it is lower

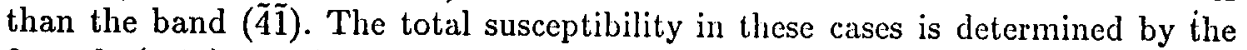
formula (7.10) as well.

If the chemical potential moves inside the band with the change of the field, then the electron concentration (average occupancy of states) in layer changes (see Fig. 5a,b). In this case the contribution of the electron subsystem into the susceptibility appears. The character of this contribution depends on the relation of the signs of parameters $d_{\mathbf{s}}$ and $d_{\mathrm{e}}$ (see Fig. $5 \mathrm{c}, \mathrm{d}$ ). Besides, the additional contributions in the pseudospin component of susceptibility $\chi_{\perp}^{s}$ appear. These contributions are caused by the change of average occupancies $\left\langle\bar{X}^{r r}\right\rangle$ influenced by the field. The latter leads to an increase in $\chi_{\perp}^{\mathrm{s}}$ for the opposite signs of $d_{\mathrm{s}}$ and $d_{\mathrm{e}}$ and to decrease in $\chi_{\perp}^{\mathbf{s}}$ for the same signs. It is caused by the fact that the change of the electron concentration in the layer influenced by the external electric field leads to the change of the effective field acting on the pseudospin by the value of $g$ for each additional electron at a site.

Such a physical picture remains also in the case when the chemical potential is in the permitted band only for the certain interval of $h$ values (Fig. 6a,b). Namely the great values of the susceptibility $\chi_{\perp}^{\text {total }}$ correspond to this interval, while outside of this interval the susceptibility is small (Fig. 6c,d).

The obtained results testify to the conclusion that certain intervals of double-well asymmetry parameter $h$ correspond to the essential increase in the transverse (i.e. normal to the layers) dielectric susceptibility. The ratio of contributions of pseudospin and electron components depends, first of all, on the relation between $d_{\mathrm{s}}$ and $d_{\mathrm{e}}$. The situation in $\mathrm{YBaCuO}$ crystal corresponds to the case when $d_{\mathrm{s}}$ and $d_{\mathrm{e}}$ have the same signs (which is connected with the increase in electron level energy in the layer when the apex oxygen is located in $S^{z}=+1 / 2$ position, that is, closer to the layer). By the order of magnitude $d_{\mathrm{s}} \simeq Z\left(\mathrm{O}_{\mathrm{A}}\right) \delta, d_{\mathrm{e}} \simeq e D$, where $Z\left(\mathrm{O}_{\mathrm{A}}\right)$ is the effective charge of $O_{I V}$ oxygen, $\delta$ is the distance between the double-well minima, and $D$ is the effective length corresponding to the transfer of the electron charge from $\mathrm{CuO}$ chains to the layers.

The pseudospin (lattice) component of the susceptibility (7.10) reaches the value of the order of $\chi_{\mathrm{s}} \simeq d_{\mathrm{s}}^{2} /\left(v_{\mathrm{c}} \Omega\right)$ at its maximum. As for the electron component $\chi_{\mathrm{e}}$, it can be evaluated as $\chi_{\mathrm{e}} \simeq d_{\mathrm{e}}^{2} /\left(v_{\mathrm{c}} \widetilde{W}\right)$ (here $\widetilde{W}$ is the subband width renormalized due to the narrowing effect), if the leading contribution

$$
\chi_{\mathrm{e}} \simeq \frac{d_{\mathrm{e}}^{2}}{v_{\mathrm{c}}} \sum_{r} n_{r} \frac{\mathrm{d}\left\langle X^{r r}+X^{\tilde{r} \tilde{r}}\right\rangle}{\mathrm{d} \mu}
$$

is extracted. Hence, the estimate $\chi_{\mathrm{e}} / \chi_{\mathrm{s}} \simeq\left(d_{\mathrm{e}}^{2} / d_{\mathrm{s}}^{2}\right)(\Omega / \widetilde{W})$ follows. Because $\delta \ll D\left(\delta \simeq 10^{-1} \AA[5], D \simeq 5 \div 7 \AA\right), \chi_{\mathrm{e}} \gg \chi_{\mathrm{s}}$ for $\Omega \leq \widetilde{W}$. Direct numeri- 


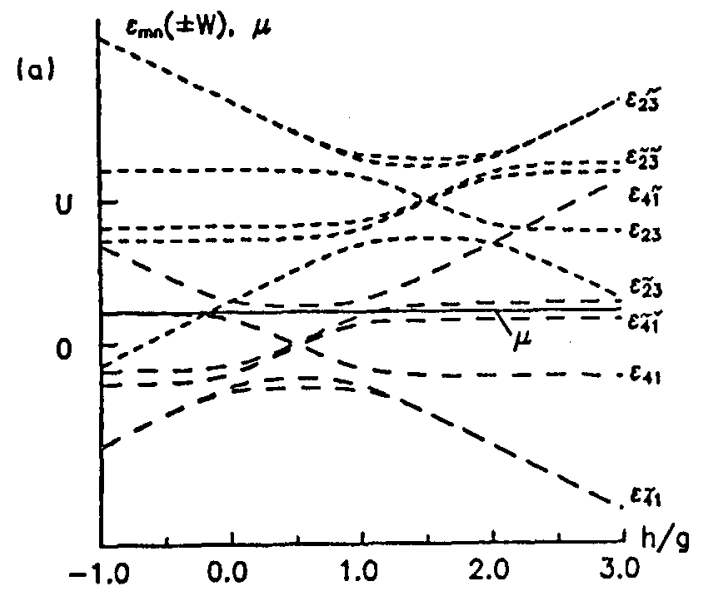

(b)
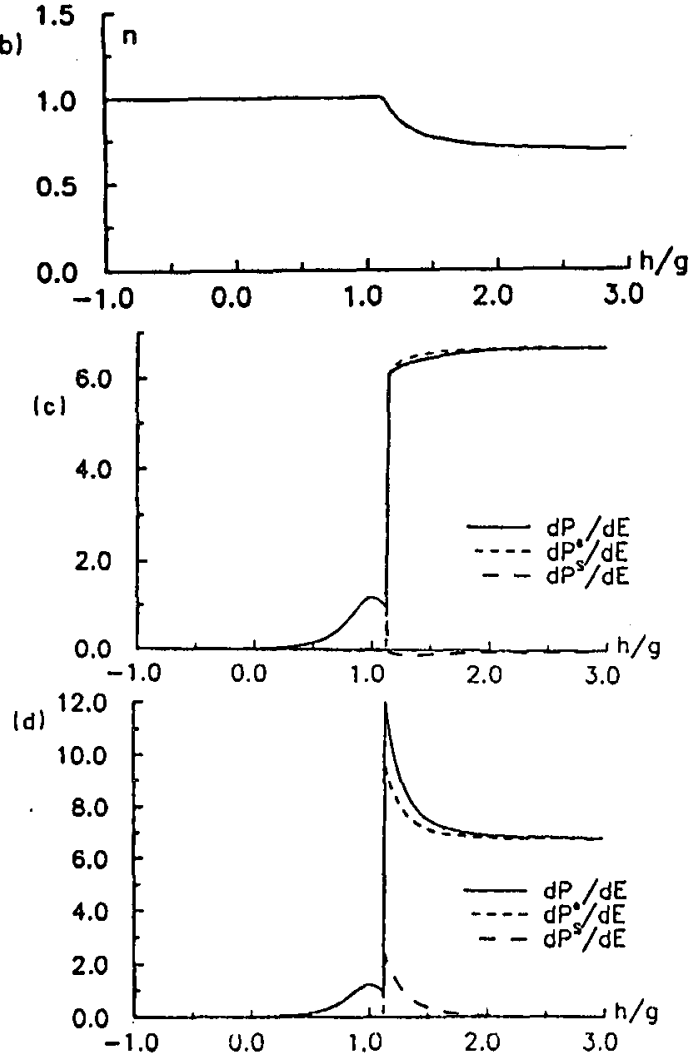

Fig. 5. Behaviour of the band picture (a), electron concentration in layer (b) and the total, electron and pseudospin susceptibilities (c, d) depending on $h / g$ $(U=2.2, g=1, h=0.7, \Omega=0.3, W=0.2, \mu=0.5$; Figures $5 \mathrm{c}$ and $5 \mathrm{~d}$ correspond to $d_{\mathrm{e}}=1, d_{\mathrm{s}}=0.6$ and $d_{\mathrm{e}}=1, d_{s}=-0.6$, respectively). 

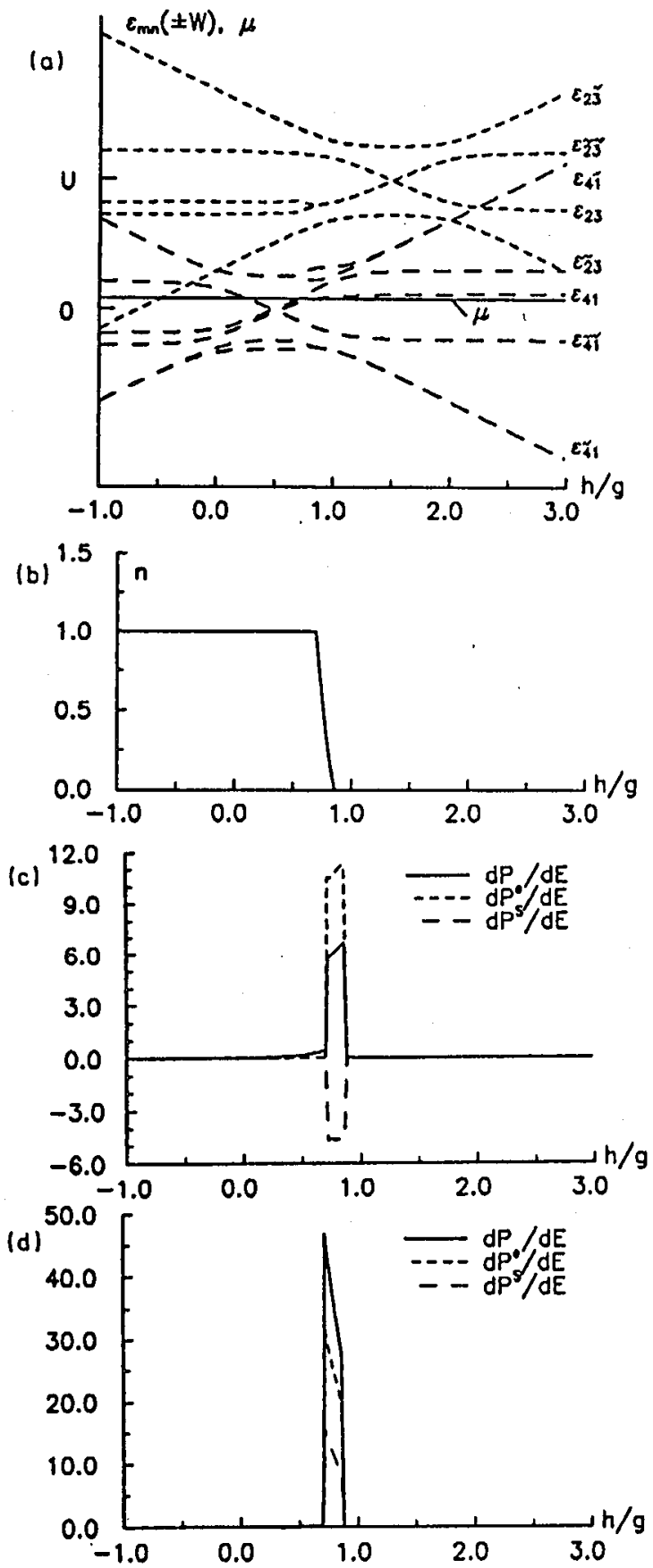

Fig. 6. The same as in Fig. 5 with $\mu=0.2$. 
cal estimation gives $\chi_{\mathrm{e}} \simeq 10^{1} \div 10^{2}$, which can match to the large observed $\varepsilon_{z z}$ permeability values $[13,14]$ and testifies to the existing tendency to the dielectric instability.

\section{Conclusion}

Thus, we have shown that the single-clectron spectrum of the Müller model displays a more complicated structure than that of the Ilubbard model. The interaction of electrons with the anharmonic vibration mode leads to the existence of extra subbands and causes their additional narrowing. The violation of electron-hole symmetry takes place. The effective exchange between electrons depends on the state of the vibrational subsystem and may possess both antifcrro- and ferromagnetic character. This could be one of the reasons for the frustration of the exchange interaction. The electron contribution to the transverse dielectric susceptibility of the model, arising from the electron charge transfer from/to CuO layer, can be of a significant magnitude leading to the high values of the static diclectric susceptibility.

\section{References}

[1] R.E. Cohen, W.E. Pickett, H. Krakauer, Phys Rev. Lelt. 64, 2575 (1990).

[2] H. Maruyama, T. Ishii, N. Bamba, II. Macda, A. Koizumi, Y. Yoslikawa, II. Yamazaki, Physica C 1C0, 524 (1989).

[3] S.D. Conradson, I.D. Raistrick, Science 243, 1340 (1989).

[4] G.H. Kwei, A.C. Larson , W.L. Hults, G.L. Smith, Physica C 169, 217 (1990).

[5] S.D. Conradson, I.D. Raistrick, A.R. Bishop, Science 248, 1394 (1990).

[6] Y. Olta, T. Tohyama, S. Maekawa, Plyysica C 166, 385 (1990).

[7] A.R. Bishop, R.L. Martin, K.A. Muller, Z. Tesanovic, Z. Phys. B, Condens. Maller 76, 17 (1989).

[8] N.M. Plakida, Phys. Scri. 29, 77 (1989).

[9] N.M. Plakida, V.L. Akscnov, S.L. Dreclisler, Europhys. Lett. 4, 1309 (1989).

[10] S.K. Kurtz, J.R. Hardy, J.W. Flocken, Ferroelectrics 87, 29 (1988).

[11] A. Bussman-Holder, A. Simon, II. Buttner, Phys. Rev. B 39, 207 (1989).

[12] V. Müller, C. Hucho, D. Maurer, Ferroclectrics 130, 45 (1992).

[13] F.M. Müller, S.P. Chen, M.L. Prueitt, J.F. Smith, J.L. Smith, D. Wohlleben, Phys. Rev. B 37, 5837 (1988).

[14] L.R. Testardi, W.G. Moulton, II. Matias, II.K. Ng, C.M. Rey, Phys. Rev. B 37, 2324 (1988).

[15] K.A. Müller, Phase Transilions (special issue) (1988).

[16] J.E. Hirsch, S. Tang, Phys. Rev. B 40, 2179 (1989).

[17] M. Frick, W. von der Linden, I. Morgenstern, II. Raedt, Z. Phys. B, Condens. Matter 81, 327 (1990).

[18] Yu.A. Izyumov, Sov. Phys.-Usp. 101, 1 (1991).

[19] P.W. Anderson, G. Baskaran, Z. Zou, Phys. Rev. Lelt. 58, 2790 (1987). 
[20] I.V. Stasyuk, A.M. Shvaika, Preprint Inst. Cond. Matt. Phys. Ukr. Acad. Sci., ICMP-91-56P, Lviv 1991; I.V. Stasyuk, A.M. Shvaika, E. Schachinger, Physica $C$ (in press).

[21] N.M. Plakida, V.S. Udovenko, Mod. Phys. Lelt. B G, 541 (1992).

[22] J. Hubbard, Proc. R. Soc. Lond. A 276, 238 (1963).

[23] A.V. Harris, R.V. Lange, Phys. Rev. 157, 295 (1967).

[24] J.E. Hirsch, Phys. Rev. Lett. 54, 1317 (1985).

[25] L.D. Didukh, I.V. Stasyuk, Ukr. Fiz. Zh. 13, 899 (1968).

[26] I.V. Stasyuk, A.M. Shvaika, Preprint Inst. Cond. Matt. Phys. Ukr. Acad. Sci., ICMP-92-15P, Lviv 1992.

[27] Lu. Y. Patton, J. Phys, Condens. Maller 2, 9423 (1990).

[28] D.N. Aristov, S.V. Maleyev, Z. Phys. B 81, 433 (1990). 